\title{
Terahertz Wave Antireflection Filter Using Nanostructure Multilayers
}

\author{
Wang Wenliang and Rong Xiaohong
}

\begin{abstract}
Over the past decades, there have been significant advances in techniques to generate and detect terahertz (THZ) signals, but there have been comparatively few reports of structures that manipulate and control them. In this paper, based on the characteristic matrix method, hydrogenated amorphous silicon (a-Si[H]) and silicon oxide (SiO2) were chosen as coating materials, a nanostructure multilayers as broadband antireflection coating used at terahertz frequencies region was designed. Which has a residual reflectivity of less than 0.08 and average reflectivity about 0.06 throughout the $50-140 \mathrm{~cm}-1$ region. But we found that the increasing of incident angle will large the average residual reflectivity and enhance the polarization-dependent deviation effect especially. Then we designed a depolarizing broadband antireflection by adopting a silicon-air multiilayer structure. It's residual reflectivity was small and corredponding polarizion effect can also be neglected.
\end{abstract}

Index Terms-Nanostructure multilayers; broadband antireflection; terahertz frequencies region

\section{INTRODUCTION}

The frequency of terahertz $(\mathrm{THz})$ radiation lies in the boundary region between light and radio waves. Customarily, the region was defined as $\mathrm{f}=0.3-10 \mathrm{THz}, \lambda=$ $1000 \mathrm{~nm}-30 \mu \mathrm{m}$. Over the past decades, there have been tremendous advances in the generation, manipulation, and detection of terahertz radiation [1-3]. All these new technologies have inspired exciting new applications for terahertz sensing and imaging due to its nonionizing nature and the ability to penetrate through most textiles currently in use[4,5]. While there have been significant advances in techniques to generate and detect terahertz signals, there have been comparatively few reports of structures that manipulate and control them. A range of terahertz components are required to manipulate the propagation of terahertz wave, inasmuch as optical components are required to control visible light, infrared, or ultraviolet. These components may comprise lenses, mirrors, parabolic mirrors, beam splitters, filters, polarisers, and so on. Most of the terahertz wave components directly adopt principles from optics. This is possible since the terahertz waves are quasi-optical. Typically, these components cause reflections due to the refractive index mismatch at interfaces giving rise to unwanted Fabry-Pérot behavior. In such cases, antireflection coatings that operate at terahertz frequencies would be highly advantageous to minimize etalon reflections[6-8].

Manuscript received May 21, 2011; revised May 29, 2011

Wang Wenliang is with the Department of Physics, Nanchang University, China.

Rong Xiaohong is with the Institute of Industry Technology, China.
In this paper, we report a nanostructure multiplayer as broadband antireflection coating used at terahertz frequencies region. The whole multiplayer stack was composed of four layers of dielectric material, and which was adhered on one side of a single-crystal germanium substrate. Then we analyze the effect about the change of incident angle and find that the polarizing effect shows more and stronger as the increasing of incident angle. To reduce the polarizing effect, we designed a multilayer nanostructure which comprises silicon wafers and dry air-ring. The two materials were fitted together by a high density polyethylene frame.

The organization of this paper is as follows. In Sec. II, we describe the characteristic matrix method, which is required to analyses the response of a multiplayer structure. The result of design, nanostructure multilayer as broadband antireflection coating used at terahertz frequencies region is given in Sec. III. Finally, a conclusion is given in Sec. IV.

\section{THEORY}

For decades, FTIR (Fourier transform infrared) spectroscopy has been widely employed in the study of materials in the frequency range around the mid-infrared. Correspondingly, multiplayer interference structures operative in this frequency range, made from a number of different materials and configurations, are widely available. Provided there is no absorption, the structure can be operated either as a filter or mirror dependent on the alignment, as the two functions are complementary. Some multilayer structures used as FTIR mirrors are, for example: zinc sulphide/polyethylene[9], silicon - air[10].

Similar multilayer structures are designed for T-rays, i.e. from 0.1 to $1.0 \mathrm{THz}$, and characterised by THz-TDS. A series of these designs are, for example: StyroluxTM/PE and polyethylene/air, polypropylene/silicon, alumina/aluminazirconia, polypropylene/polypropylene+TiO2 . These earlier studies focus on finding materials and fabrication techniques suitable for the operation of the multilayer structures in the T-ray frequency range. The fabricated structures are expected to be used as mirrors for short-range or indoor Tray communication. Thus, particular interest is given to an economic material fabrication, which provides a structure with the highest reflectivity and broadest reflection band at any angle of incidence.

A multilayer interference filter is composed of several dielectric layers with different indices of refraction. The following subsection briefly discusses the characteristic matrix method, which is required to analyses the response of a multilayer structure [11]. In order to analyze the response of the described structure, the characteristic matrix method is engaged. In brief, a characteristic matrix for high-indexed material, in the case that the wave propagation direction is 
parallel with the stacking direction, is given by

$$
M_{H}=\left[\begin{array}{cc}
\cos \left(\frac{\omega}{c} n_{H} l_{H}\right) & j \sin \left(\frac{\omega}{c} n_{H} l_{H}\right) / n_{H} \\
j n_{H} \sin \left(\frac{\omega}{c} n_{H} l_{H}\right) & \cos \left(\frac{\omega}{c} n_{H} l_{H}\right)
\end{array}\right]
$$

where $n_{H}$ and $l_{H}$ are the refractive index and the thickness attributed to a high-indexed material. Likewise, a matrix for the low-indexed material is

$$
M_{l}=\left[\begin{array}{cc}
\cos \left(\frac{\omega}{c} n_{L} l_{L}\right) & j \sin \left(\frac{\omega}{c} n_{L} l_{L}\right) / n_{L} \\
j n_{L} \sin \left(\frac{\omega}{c} n_{L} l_{L}\right) & \cos \left(\frac{\omega}{c} n_{L} l_{L}\right)
\end{array}\right]
$$

Here $n_{L}$ and $l_{L}$ are the refractive index and the thickness attributed to a low-indexed material. When slabs of these materials are layered using a periods, with the highindexed material terminating both ends, the resulting characteristic matrix is simply obtainable via matrix multiplications in the proper order:

$$
M_{\text {total }}=\left[\begin{array}{ll}
m_{11} & m_{12} \\
m_{21} & m_{22}
\end{array}\right]=\left(M_{H} M_{L}\right)^{\alpha} M_{H}
$$

The transmission function of the structure in free space is calculated from the total characteristic matrix:

$$
T(\omega)=\frac{2}{m_{11}+m_{12}+m_{21}+m_{22}}
$$

This transmission function, $T(\omega)$, is related to the transmittance, $\Gamma(\omega)=|\mathrm{T}(\omega)|^{2}$.

When terahertz wave at oblique incidence on multilayer structure, the incidence angles on each interface were related by Snell's law

$$
n_{0} \sin \theta_{0}=n_{H} \sin \theta_{H}=n_{L} \sin \theta_{L}=n_{S} \sin \theta_{S}
$$

where $n_{0}, n_{H}, n_{L}$ and $n_{S}$ were the refractive index of incident media, high-index, low-index and substrate material respectively. $\theta_{0}, \theta_{H}, \theta_{L}$ and $\theta_{S}$ corresponding to the angle of terahertz wave in various materials as shown in Fig.1.

Based on the theory of electromagnetic wave transmission, in general, plane wave at oblique incidence on a dielectric material will have transverse electric(TE) and transverse magnetic(TM) fields respectively. They will experience different reflectances $R_{T E}$ and $R_{T M}$ or transmission $T_{T E}$ and $T_{T M}$. Such polarization-dependent properties were inherent and intolerable in many applications.

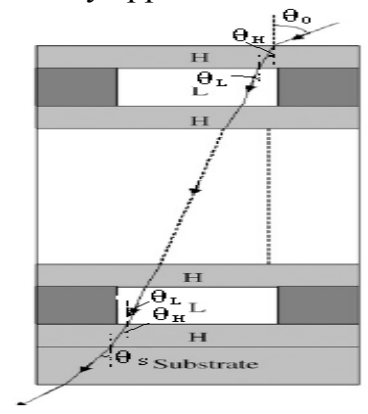

Fig.1 Schematic diagram of the terahertz wave transimssion at oblique incidence on multilayer structure

The tangential parts of TE and TM should keep consecutive when electromagnetic wave transmits at the interface of multilayers. This physical essence leaded to TE and TM show differen equivalent index

$$
\left\{\begin{array}{l}
n_{T M}=\frac{n}{\cos \theta} \\
n_{T E}=n \cos \theta
\end{array}\right.
$$

So $n_{H}, n_{L}, n_{0}$ and $n_{S}$ in equation (1), (2) and (5) should be substituted by

$$
\left\{\begin{array}{l}
n_{H, T M}=\frac{n_{H}}{\cos \theta_{H}} \\
n_{L, T M}=\frac{n_{L}}{\cos \theta_{L}} \\
n_{0, T M}=\frac{n_{0}}{\cos \theta_{0}} \\
n_{S, T M}=\frac{n_{S}}{\cos \theta_{S}}
\end{array}\right.
$$

for TM field, and

$$
\left\{\begin{array}{l}
n_{H, T E}=n_{H} \cos \theta_{H} \\
n_{L, T E}=n_{L} \cos \theta_{L} \\
n_{0, T E}=n_{0} \cos \theta_{0} \\
n_{S, T E}=n_{S} \cos \theta_{S}
\end{array}\right.
$$

for TE field respectively. Then strong polarization effects (PE) can be gained from equation (4) in theory.

\section{Design AND Result}

In the design of coating, Hydrogenated amorphous silicon (a-Si[H]) and silicon oxide ( $\mathrm{SiO} 2)$ were chosen as coating materials because: the ratio of refractive indices of the two materials is relatively large $(\mathrm{a}-\mathrm{Si}[\mathrm{H}] \mathrm{n}=3.7-2.8 ; \mathrm{SiO} 2 \mathrm{n}=2)$ and the extinction coefficients of these materials at terahertz frequencies are not so large (10-2).

As shown in Fig.2, the optical constants of the materials that were used in our design can be found in corresponding literatures[12].

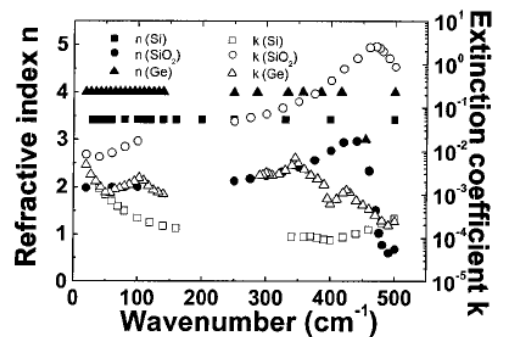

Fig.2 The optical constants of the materials that have been used in the design

From which, we can find that the refractive index of c-Si remains constant at 3.42 throughout the plotted region and there is a gradual increase in the refractive index of $\mathrm{SiO} 2$ from $1.96(20 \mathrm{~cm}-1)$ to 2.94 (at $440 \mathrm{~cm}-1)$ with an increase in wavenumber.

Based on these data and the characteristic matrix method which have be shown in section $\Pi$, an antireflection coating structure consisting of four layers was designed for using in terahertz frequencies region. The nanostructure multilayers were adhered on one side of a single-crystal germanium (Ge) substrute. The structure of whole system is Ge Substrate/Si $8.23 \mu \mathrm{m} / \mathrm{SiO} 23.71 \mu \mathrm{m} / \mathrm{Si} 2.32 \mu \mathrm{m} / \mathrm{SiO} 215.25 \mu \mathrm{m} / \mathrm{Air}$, which has be shown in Fig.3. Corresponding antireflection for design structure has be shown in Fig. 4, and we can find it has a residual reflectivity of less than 0.08 and average 
reflectivity about 0.06 throughout the $50-140 \mathrm{~cm}^{-1}$ region.

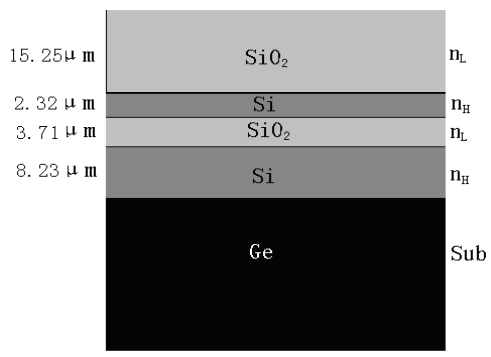

Fig.3 Schematic of nanostructure multiplayers for antireflection in terahertz frequencies

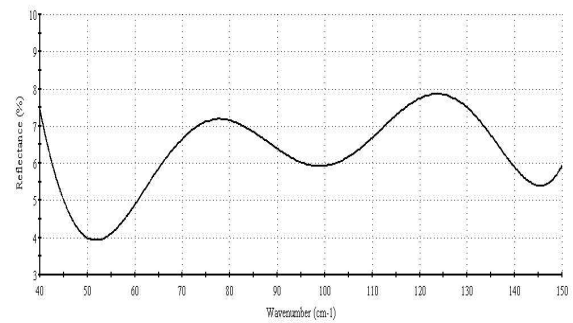

Fig.4 Calculated antireflection for designed structure: Ge Substrate/Si 8.23 $\mu \mathrm{m} / \mathrm{SiO} 23.71 \mu \mathrm{m} / \mathrm{Si} 2.32 \mu \mathrm{m} / \mathrm{SiO} 215.25 \mu \mathrm{m} /$ Air using characteristic matrix method

Then we analysed the effect of incidence angle change on spectrum of this design. Fig.5(a-d) showed the spectral response of the TE and TM polarization, when the incidence angle equals to $5^{\circ}, 15^{\circ}, 30^{\circ}$ and $45^{\circ}$, respectively.

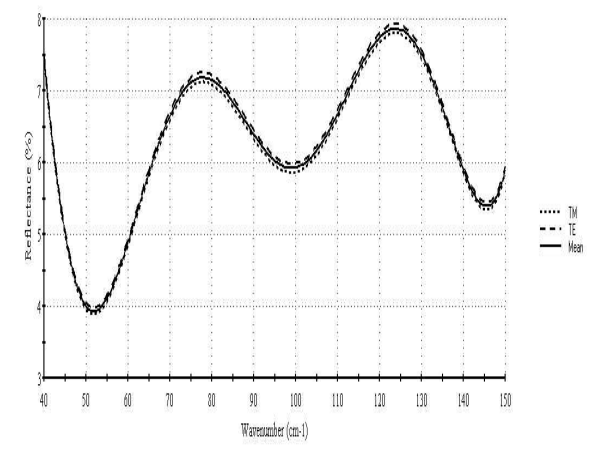

(a)

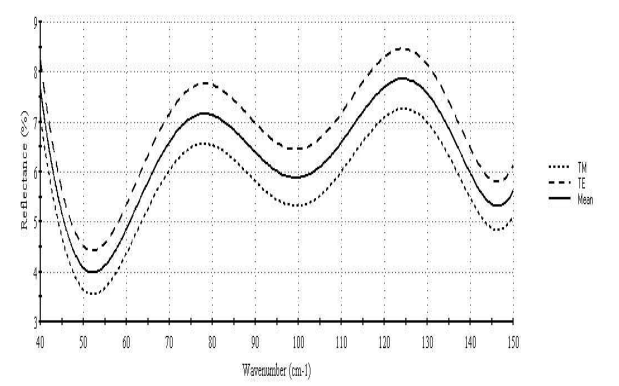

(b)

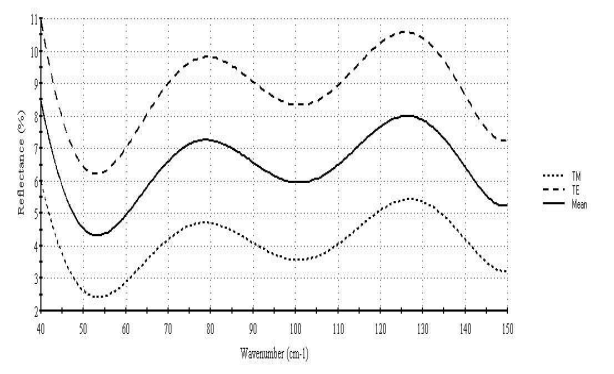

(c)

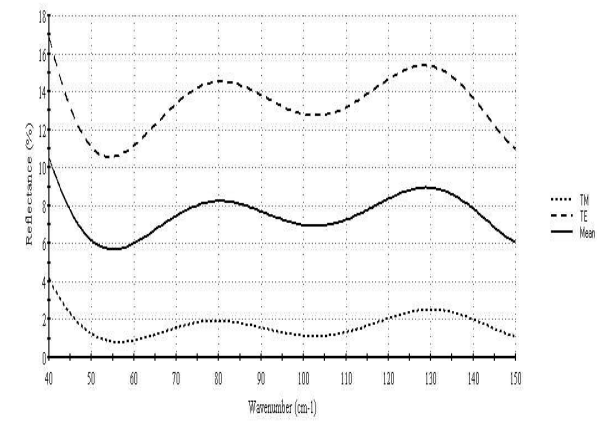

(d)

Fig.5 TE and TM reflection spectrum of the proposed multilayer nanostructure as shown in Fig. 3 with different incident angle. (a) $5 \mathrm{o}$; (b) $15 \mathrm{o}$; (c) $30 \mathrm{o}$; (d) $45 \mathrm{o}$

For the case of 50 incidence angle, Fig.5 (a) showed that the deviation between the reflection of TE and TM fields was very small and can be neglected. But with the increasing of incident angle, polarization effect became more and more strong. Fig.5(b) showed that the deviation between the reflection of TE and TM fields with incident angle 150 was already very evident. From Fig.5(c), we can find that the average residual reflectivity with incident angle 30 o was almost the same as the case of vertial incident, but the deviation between the reflection of TE and TM fields reach to about 0.06 . From Fig.5(d), we can find that not only the deviation between the reflection of TE and TM fields with incident angle 450 even reach 0.14 , but the average residual reflectivity also increased to about 0.08 .

So we can say that the increasing of incident angle will make the spectral character of the design bad, including the largen of average residual reflectivity and the enhancement of polarization-dependent deviation effect especially. To design depolarizing broadband antireflection is essential to terahertz wave in oblique incidence. But this work was very difficult because the polarization effect was inhered to single layer dielectric materials.

One method to reduce polarization effect was adopting a multilayer structure with appropriate materials combinations. Silicon and dry air were selected as materials for construction of the depolarizing broadband antireflection operating at terahertz region in our paper. Silicon material was known to have a negligible absorption, low dispersion and a constant refractive index of 3.418 in terahertz regime. Dry air has a unity refractive index, nearly zero absorption and dispersion.

The difference of refractive index between silicon and air was large; it was advantageous factor for reducing polarizing effect in our design.

Then proper material arrangement should be designed to achieve required spectrum character on terahertz wave region. A multilayer structure, comprising silicon layer and air layer, was an attractive option because of its structural simplicity yet optical functionality. Air layer was created by open rings and silicon layer as shown in Fig.6. The two materials were fitted together by a high density polyethylene frame. 


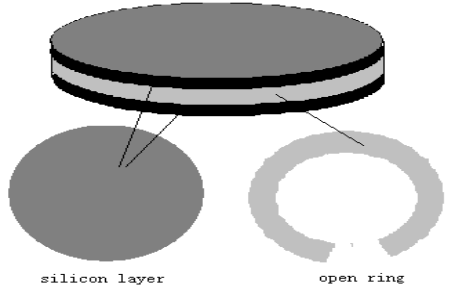

Fig.6 Schematic of air layer

As shown in Fig.7, a depolarizing antireflection coating structure consisting of four air layers and five silicon layers was designed for using in terahertz frequencies region. The multilayers structure was adhered on one side of a singlecrystal germanium $(\mathrm{Ge})$ substrute. The structure of whole system was Ge Substrate/Si $9.36 \mu \mathrm{m} /$ air $3.50 \mu \mathrm{m} / \mathrm{Si} 3.97 \mu$ $\mathrm{m} /$ air $8.36 \mu \mathrm{m} / \mathrm{Si} 2.49 \mu \mathrm{m} /$ air $14.54 \mu \mathrm{m} / \mathrm{Si} 1.36 \mu \mathrm{m} / 21.26$ $\mu \mathrm{m} / \mathrm{Si} 0.55 \mathrm{~m} / \mathrm{Air}$.

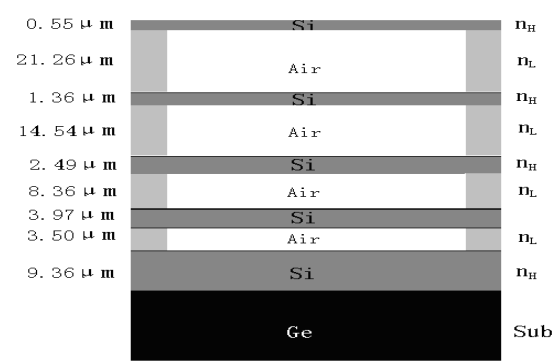

Fig.7 Schematic of silicon-air multilayer structure for depolarizing broadband antireflection in terahertz frequencies region

Based on characteristic matrix theory which have be shown in section $\Pi$, we showed the corresponding TE and TM reflection spectrum of the proposed silicon-air multilayer structure as shown in Fig.7 with incident angle $45^{\circ}$. We can find it has a residual reflectivity of less than $0.8 \%$ and average reflectivity about $0.25 \%$ throughout the $50-140 \mathrm{~cm}^{-1}$ region. The average deviation between the reflection of TE and TM fields was about $0.3 \%$. The residual reflectivity of this multilayer structure was small and corresponding polarizing effect can be neglected.

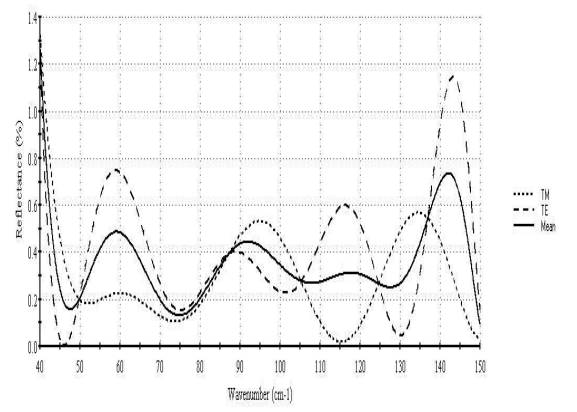

Fig.8 TE and TM reflection spectrum of the proposed silicon-air multilayer structure as shown in Fig. 7 with incident angle $45^{\circ}$.

\section{CONClusion}

In conclusion, we have achieved a nanostructure multilayer as broadband antireflection used at terahertz frequencies region. In our design, we choose hydrogenated amorphous silicon and silicon oxide as high and low refractive index coating materials respectively for their large refractive index ratio and small extinction coefficients at terahertz frequencies region. The whole multilayer stack was adhered on one side of a single-crystal germanium substrate. The results of our design shows that the structure has a residual reflectivity of less than 0.08 and average reflectivity about 0.06 throughout the $50-140 \mathrm{~cm}-1$ region. Then we found that the increasing of incident angle will make the spectral character of the design bad, including the largen of average residual reflectivity and the enhancement of polarization-dependent deviation effect especially. We designed a depolarizing broadband antireflection by adopting a silicon-air multiilayer structure. Its residual reflectivity was small and corredponding polarizion effect can be neglected.

\section{REFERENCES}

[1] D. M. Mittleman, M. Gupta, R. Neelamani, R. G. Baraniuk, J. V. Rudd, and M. Koch, "Recent advances in terahertz imaging," Appl. Phys. B 68(6), 1085-1094 (1999).

[2] R. M. Woodward, B. E. Cole, V. P. Wallace, R. J. Pye, D. D. Arnone, E. H. Linfield, and M. Pepper, "Terahertz pulse imaging in reflection geometry of human skin cancer and skin tissue," Phys. Med. Biol. 47(21), 3853-3863(2002).

[3] D. Grbovic, and G. Karunasiri, "Fabrication of Bi-material MEMS detector arrays for THz imaging," Proc. SPIE, (2009), p. 731108.

[4] N. I. Landy, C. M. Bingham, T. Tyler, et al. "Design, theory, and measurement of a polarization-insensitive absorber for terahertz imaging", Phys. Rev. B 79(12), 125104(2009).

[5] Edwin J. Heilweil, James E. Maslar, William A. Kimes, et al., "Characterization of metal oxide nanofilm morphologyies and composition by terahertz transmission spectroscopy", Optics Letters. 34(9), 1360-1362(2009).

[6] J. Kröll, J. Darmo, and K. Unterrainer, "Metallic wave-impedance matching layers for broadband terahertz optical systems",Opt. Express 15, 6552(2007).

[7] J. Kröll, J. Darmo, and K. Unterrainer, Electron. Lett. 40, 763(2004).

[8] Andreas Thoman, Andreas Kern, Hanspeter Helm, and Markus Walther, "Nanostructured gold films as broadband terahertz antireflection coatings", Phys. Rev. B 77(19), 195405(2008).

[9] J. Shao, J.A. Dobrowolski, "Multilayer interference filters for the farinfraredand submillimeter regions" Applied Optics 32 (13), 23612370 (1993)

[10] R. Schiwon, G. Schwaab, E. Bru"ndermann, M. Havenith, Applied Physics Letters 83 (20) (2003) 4119.

[11] Withawat Withayachumnankul, Bernd M. Fischer, Derek Abbott, "Quarter-wavelength multiplayer interference filter for terahertz waves", Optics Communications, 281, 2374-2379(2008).

[12] E. D. Palik, ed., Handbook of Optical Constants of Solids (Academic, Orlando, Fla., 1985), pp. 465-478, 547-569, 749-763.

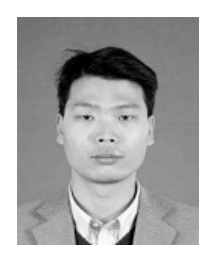

Wang Wenliang, was born on August 28, 1978 in Hangzhou, China. He earned Bachelor and Master degree in the Department of Physics in Sichuan Normal University in 2002 and 2005 respectively. Then in 2008, he earned Doctor degree in the Institute of Optics and Electronics, Chinese Academy of Sciences. Currently he is a instructor in Physical department of Nanchang University. His research is mainly focused on optical coating and film design. 